\title{
Eurostudia
}

\section{Repenser l'histoire des médias à partir du référent balzacien dans Verlorene Illusionen de Helmut Wietz}

\section{Louise-Hélène Filion}

Volume 13, numéro 1-2, 2018-2019

Cultures en contact, entre régulations et représentations

URI : https://id.erudit.org/iderudit/1067284ar

DOI : https://doi.org/10.7202/1067284ar

Aller au sommaire du numéro

\section{Éditeur(s)}

Le Centre canadien d'études allemandes et européennes

ISSN

1718-8946 (numérique)

Découvrir la revue

Citer cet article

Filion, L.-H. (2018). Repenser l'histoire des médias à partir du référent balzacien dans Verlorene Illusionen de Helmut Wietz. Eurostudia, 13(1-2), 273-297. https://doi.org/10.7202/1067284ar
Résumé de l'article

Dans le roman graphique Verlorene Illusionen (2017), Helmut Wietz s'inspire librement d'Illusions perdues de Balzac, mais il déplace la satire des milieux littéraires et journalistiques parisiens dans le contexte de l'Allemagne divisée. L'Allemagne sur le chemin de la réunification de 1989-1990, et celle de la République démocratique allemande des années 1969-1970 imaginées par Wietz partagent toutefois des traits du monde hypercontemporain, le bédéiste s'appuyant sur l'anachronie pour mener une critique sur l'emprise des médias et leur mise en images du réel; pour déplorer l'instrumentalisation de l'information, de la littérature et des mots. Cet article présente les modalités de l'appropriation de l'oeuvre balzacienne dans le contexte d'une réécriture de l'histoire des médias en RFA-RDA; l'examen de l'emploi par Wietz des ressources propres au médium de la bande dessinée - les choix narratifs, l'organisation du lettrage et des lieux, etc. - sera également éclairant quant à la nature du traitement wietzéen de l’Histoire.
Tous droits réservés $@$ Le Centre canadien d'études allemandes et européennes, 2019
Ce document est protégé par la loi sur le droit d'auteur. L'utilisation des services d’Érudit (y compris la reproduction) est assujettie à sa politique d'utilisation que vous pouvez consulter en ligne.

https://apropos.erudit.org/fr/usagers/politique-dutilisation/ 


\section{Repenser l'histoire des médias à partir du référent balzacien dans Verlorene Illusionen de Helmut Wietz}

\section{Louise-Hélène Filion \\ Université du Michigan}

\section{Résumé}

Dans le roman graphique Verlorene Illusionen (2017), Helmut Wietz s'inspire librement d'Illusions perdues de Balzac, mais il déplace la satire des milieux littéraires et journalistiques parisiens dans le contexte de l'Allemagne divisée. L'Allemagne sur le chemin de la réunification de 1989-1990, et celle de la République démocratique allemande des années 1969-1970 imaginées par Wietz partagent toutefois des traits du monde hypercontemporain, le bédéiste s'appuyant sur l'anachronie pour mener une critique sur l'emprise des médias et leur mise en images du réel; pour déplorer l'instrumentalisation de l'information, de la littérature et des mots. Cet article présente les modalités de l'appropriation de l'œuvre balzacienne dans le contexte d'une réécriture de l'histoire des médias en RFA-RDA; l'examen de l'emploi par Wietz des ressources propres au médium de la bande dessinée - les choix narratifs, l'organisation du lettrage et des lieux, etc. - sera également éclairant quant à la nature du traitement wietzéen de l'Histoire.

\section{Abstract}

Helmut Wietz's graphic novel Verlorene Illusionen (2017) is loosely based on Illusions perdues, but Balzac's satire of Parisian literary and journalistic circles has been relocated to divided Germany. Wietz's imagined Germany, which includes Germany on the road to 
reunification in 1989-1990 and the German Democratic Republic in 1969-1970, is in some ways utterly contemporary: anachronistically, the artist criticizes the media's hold on us as they transform reality into images and regrets the instrumentalization of information, literature and words. This article examines how Wietz appropriates Balzac's work in rewriting the history of the media in West and East Germany. A focus on his use of resources specific to comic artnarrative choices, the organization of the lettering and of space, etc. - also helps us understand his view of history.

Dès la première page de Verlorene Illusionen, on aperçoit clairement le dramaturge est-allemand Lucien, incarnation graphique du fameux Lucien de Rubempré de Balzac, qui traverse le mur de Berlin pour fuir illégalement à l'Ouest, taraudé d'ambitions littéraires et désireux de pouvoir montrer, maintenant qu'il est passé à l'Ouest, « was für ein großartiger Theaterautor ich bin!»1 (Wietz 2017 : 5; ci-après VI). Les soldats au poste-frontière, se demandant s'ils doivent l'abattre, décident de ne pas passer à l'acte, et choisissent de le laisser circuler librement: "Nee, lass mal. Ab morgen gibt's die DDR sowieso nicht mehr! »" $(V I: 5)$. Le ton est donné, dès la première page de ce roman graphique paru en 2017; il s'agit pour Helmut Wietz de mettre en scène de manière extrêmement libre l'histoire allemande à partir du référent balzacien qui a servi de matrice à la composition du texte, s'autorisant les invraisemblances et les anachronies, confondant ultimement les époques, mais toujours à partir d'une ligne interprétative claire: la réécriture de l'Histoire est ancrée dans une profonde satire des milieux de l'art et des médias, ainsi que du rapport à la technologie. En

\footnotetext{
1 «quel dramaturge formidable je suis! » Toutes les traductions que comporte cet article sont de l'auteure.

2 « Non, laisse tomber. À partir de demain il n'y a plus de RDA, de toute manière! »
} 
se déployant à partir de "historisch-politische Inhalte »" ${ }^{3}$ cette récente œuvre s'appuie sur l'un des thèmes privilégiés du roman graphique actuel (Abel et Klein 2016 : 162). Qui plus est, Wietz ajoute à sa réécriture de l’Histoire, dont la première partie est située dans la République fédérale allemande en 1989-1990, et la seconde dans la République démocratique allemande des années 1969-1970, un horizon transculturel, en déplaçant de manière inattendue l'action de ses vignettes à Paris, ou alors en intégrant des réflexions sur des enjeux liés à la mondialisation et à la circulation internationale de la culture.

Chez Wietz, les médias d'aujourd'hui ressemblent non seulement fortement, sur les plans de leur fadeur ou de leur corruption, aux milieux artistiques et médiatiques de la RFA et de la RDA, mais ils s'apparentent également à ceux que décrivait déjà Balzac dans Illusions perdues. Afin de présenter les spécificités de la réécriture de l'Histoire proposée par Helmut Wietz dans Verlorene Illusionen, j'envisagerai d'abord les modulations de la relation intertextuelle pour établir les similitudes, mais aussi les écarts vis-à-vis du roman de Balzac; l'affiliation avec Illusions perdues se traduit d'ailleurs par la mise en scène d'un espace-temps franco-allemand dans le roman graphique, dont les représentations sont d'emblée révélatrices quant au projet wietzéen de mise en fiction de l'histoire. Puis, je me pencherai sur les principaux usages de l'anachronie dont témoigne Verlorene Illusionen, en caractérisant la portée-réseau de celle-ci par un appui sur le travail de Jacques Rancière (1996). L'examen des principales formes d'anachronie dévoile que la démarche de réécriture de l'Histoire proposée par Wietz repose avant tout sur une tension entre individualisme et rapport à la collectivité; on verra que, dans les très rares passages qui s'éloignent de la pure réécriture de l'Histoire pour privilégier une pétition d'authenticité historique, la même tension est également représentée.

3 « contenus historico-politiques ». 
Enfin, je me pencherai sur la scène finale du roman graphique, qui constitue une synthèse spectaculaire des réflexions sur le progrès technique que développe le roman graphique. Si, à l'instar de nombreuses adaptations sous forme de bande dessinée d'une grande cuvre de la littérature mondiale, le récent roman graphique Verlorene Illusionen intègre ponctuellement des réflexions d'ordre esthétique, se référant au vocabulaire des études ou de la critique littéraires (Schmitz-Emans 2012 : 14), c'est avant tout par l'appui sur les ressources propres au médium qu'il emploie - la possibilité de manipuler la cohérence du décor, les usages des transitions entre les vignettes ou des changements dans le lettrage que Helmut Wietz met pleinement l'histoire en fiction.

\section{Un « non-lieu » franco-allemand}

La première partie du roman graphique se déroule ainsi dans la RFA, en 1989-1990, sur une période d'un peu plus d'un an, pendant ce moment important de transition vers l'Allemagne réunifiée. L'action se déroule à Berlin-Ouest, mais aussi à Paris. Évoqué en introduction, le «saut» de Berlin-Est à Berlin-Ouest demeure associé, comme c'était le cas dans le passage de la province à Paris que l'on trouve dans Illusions perdues, à la possibilité de goûter à la reconnaissance littéraire. Toutefois, si le Lucien Chardon de Balzac hésite encore, lorsqu'il se rend à Paris après avoir quitté son Angoulême natale, entre le "système de pauvreté soumise que prêch[e]) le Cénacle » (Balzac 1981[1837-1843] : 288), c'està-dire la voie noble et courageuse de l'ascétisme qui le conduit, surtout au début de son séjour à Paris, à consacrer toutes ses journées à l'étude à la Bibliothèque Sainte-Geneviève, et le succès rapide par le journalisme qui cependant l'incite à vendre son âme et sa pensée, cette hésitation entre deux systèmes est nettement moins représentée dans le roman graphique.

Au même titre qu'Illusions perdues, le texte de Wietz présente le monde de l'art et les milieux qui entourent sa production et sa réception, et les mondes de la 
télévision et du journalisme télévisé représentés dans la seconde partie, comme des univers foncièrement orientés vers le profit. Cependant, son Lucien est avant tout un ambitieux, être quasi mégalomane qui ressemble davantage au Lucien de Rubempré avide d'une reconnaissance foudroyante, et acceptant de se lancer dans le journalisme pour récolter rapidement de l'argent. Les époques que décrit Wietz - la période de la réunification, les années 1960 en RDA, la période hypercontemporaine - ne réservent à peu près aucune place au sacerdoce de l'homme de lettres, de l'artiste.

Le lien avec le roman de Balzac est souligné de diverses manières; ainsi, la première splash page du roman graphique est coiffée du titre «Ein grosser Mann aus der Provinz in Westberlin! $»^{4}(V I: 10)$, manière de transformer le fameux titre de la seconde partie d'Illusions perdues: "Un grand homme de province à Paris ». Outre Lucien, d'autres personnages d'Illusions perdues et de la Comédie humaine surgissent dans le roman graphique; mentionnons notamment Étienne Lousteau, le baron de Nucingen et Esther, qui portent précisément les noms que Balzac leur avait attribués. Avec Lucien, l'autre personnage d'Illusions perdues qui occupe la place la plus importante est en définitive Nais, son prénom ne comportant toutefois pas le tréma français: Naïs constituait bien entendu le surnom de Louise de Bargeton. On sait que, chez Balzac, Naïs soutient le jeune poète Lucien dont elle est amoureuse, mais en faisant presque de lui sa chose, son phénomène littéraire : elle l'introduit ainsi dans son cercle provincial.

Nais, dans le roman graphique, n'entretient pas une relation amoureuse avec Lucien, mais elle devient bien, d'une certaine façon, sa souteneuse dans le monde du théâtre à Berlin-Ouest, vivant du gain de Lucien et lui refusant des avances sur son salaire, ayant réussi à lui soutirer les droits de ses pièces. Dans la première partie du roman graphique, un producteur de séries télévisées

\footnotetext{
4 « Un grand homme de province à Berlin-Ouest ».
} 
maintient également Lucien sous sa dépendance; tout comme ceux de Nais, ses agissements évoquent directement la manipulation dont font preuve les nombreux éditeurs et les propriétaires de journaux parisiens qu'on trouve chez Balzac, uniquement intéressés par la possibilité des ventes, et qui demandent à un Lucien de Rubempré criblé de dettes, au même titre que le Lucien de Wietz d'ailleurs, de s'engager avec eux dans le long terme, en publiant non pas son seul fameux roman sur l'histoire de la France, mais en souhaitant l'obliger à rédiger une collection d'œuvres à un rythme très rapide, dont les titres ont déjà été choisis pour lui. Bien entendu, l'on relève également des écarts plus substantiels dans la démarche de Wietz, par rapport au modèle que représente le roman de Balzac; une telle distance est parfois nécessaire pour que Wietz adapte son propos aux époques qu'il décrit. Ainsi, dans Verlorene Illusionen, Lucien devra notamment se faire scénariste, ce qu'il considère « unter meiner Würde $»^{5}$ (VI: 15), alors que c'est surtout la voie du journalisme qui était décriée par les membres du Cénacle.

Néanmoins, l'essentiel du propos balzacien sur les milieux littéraires et journalistiques est certainement reconduit : il n'y a pas de doute que Wietz a été inspiré par l'aspect totalisant de la critique balzacienne; car c'est bien le fonctionnement de tous les milieux et de tous les réseaux qui à Paris permettent à la littérature d'exister, qui se voit très minutieusement dénoncé. Balzac ne se contente toutefois pas de présenter un Lucien Chardon qui découvre que les préoccupations de chacun gravitent autour de l'argent et non de l'art, aussi bien au théâtre et à la librairie qu'au journal (Balzac 1981 [1837-1843] : 295). Ainsi, il décrit de manière tout aussi détaillée les supports matériels concrets sur lesquels la transmission des textes repose: on se souviendra, par exemple, des nombreuses explications sur la fabrication du papier. Au fil de mon analyse, il

\footnotetext{
5 « sous ma dignité ».
} 
deviendra évident qu'une réflexion sur le médium, sur l'aspect technique des dispositifs qui structurent la production artistique et journalistique, est indissociable de la démarche de Wietz, renforçant ainsi le poids de l'affiliation avec Balzac.

Dans la première partie du roman graphique, Lucien se rend fréquemment à Paris, par exemple pour y fêter la première de sa pièce « Gloire » dans un Procop à l'appellation germanisée. De ce point de vue, Wietz fait souvent l'économie du déplacement : on passe ainsi de Berlin à Paris de manière fort rapide, le bédéiste s'appuyant sur des "scene-to-scene transitions, which transport us across significant distances of time and space» (McCloud $1993: 71^{6}$ ). Il est clair que Wietz souhaite ainsi établir un lien entre les milieux artistiques de Paris, présentés comme fortement corrompus, et ceux de l'Allemagne de l'Ouest au sein desquels Lucien travaille. Un univers franco-allemand s'esquisse donc dans la bande dessinée; de ce point de vue, une page particulière doit être mentionnée. La page en question comporte trois vignettes disposées à l'horizontale, lesquelles rassemblent avant tout Lucien et le producteur qui avait tenté de le maintenir sous sa tutelle, lui ayant demandé de rédiger plusieurs scénarios pour sa nouvelle télésérie et lui ayant refusé toute forme d'honoraires, peu satisfait des textes que Lucien avait pourtant rendus. Sur la page en question, toutefois, la donne a changé : grâce à Nais dont il est néanmoins toujours dépendant, Lucien est devenu le rédacteur en chef des séries d'une nouvelle chaîne privée. Sur la première vignette de la page sont représentées, en arrière-plan, des montagnes russes, et une affiche indique : «Das größte Volksfest - Deutsch französis[troncature] Volksfe[troncature] - 14.06.28.06.1[troncature] $»^{7}(V I: 31)$. Des informations sont donc manquantes sur l'affiche, que j'ai indiquées ici par le terme «troncature » : si le lecteur complétera

\footnotetext{
${ }^{6}$ " transitions de "scène à scène", qui nous font traverser des distances significatives dans le temps et l'espace ».

7 «La plus grande fête foraine - Fê[troncature] foraine germano-françai[troncature] - 14.06.28.06.1[troncature]».
} 
aisément, de lui-même, la première troncature pour en arriver à «französisches » et la seconde pour en arriver à "Volksfest», il est en revanche plus difficile de déterminer l'année au cours de laquelle cette fête foraine a lieu, fête qui semble d'ailleurs d'une durée bien longue pour une simple foire franco-allemande. L'absence de dates crée ici une certaine confusion - est-on en 1989 ou en 1990? qui est renforcée par le fait qu'on apprend, dans la troisième vignette, que la foire a lieu à Paris; la tour Eiffel, symbole reconnaissable entre tous, ainsi qu'un joueur d'accordéon sont représentés. Or, rien ne donnait à penser, avant cette case de clôture de la fête franco-allemande, que l'action se déroule à Paris : au contraire, l'action des pages précédant la scène de la fête foraine était clairement située à Berlin-Ouest. De même, l'affiche figurant sur la vignette qui ouvre la séquence de la foire n'est rédigée qu'en allemand.

La deuxième case de la page, quant à elle, présente Lucien et le producteur qui se trouvent ensemble dans une montagne russe. Alors qu'on aurait pu penser que la réflexion ouverte par la séquence de la fête foraine soit tendue vers l'Histoire, vers un ancrage historique réel - après tout, il s'agit bien de situer résolument l'action en terrain franco-allemand et de s'appuyer sur ce qui pourrait faire figure de «lieu de mémoire », la tour Eiffel - il ressort plutôt de cette page un sentiment de désordre, non seulement attribuable aux anomalies spatiotemporelles déjà évoquées, mais aussi à la présence des montagnes russes, seul élément du décor qui soit repris dans les trois vignettes de la page. Cette omniprésence du parc d'attractions donne le sentiment d'un «non-lieu », d'après la notion développée par Marc Augé, comme si Wietz avait voulu ériger un lieu qui ne permette ni l'ancrage dans l'histoire ou dans des formes de sociabilité structurées dans le temps, ni le développement d'un rapport identitaire au lieu (Augé 1992 : 69). C'est précisément dans un tel contexte anhistorique que Lucien en viendra, pour la toute première fois dans le roman graphique, à se faire luimême abuseur, à tirer profit de sa nouvelle position de rédacteur en chef pour 
exiger du producteur un pourcentage des recettes de la série policière que celui-ci produit. Si l'univers franco-allemand mis en scène est véritablement celui de l'appât du gain, c'est aussi celui du malaise, le producteur, dans la toute dernière case, affirmant se sentir soudainement très mal - le bas de son visage est rouge sang, et des taches rouges ornent également sa chemise - alors qu'on ne sait pas si ce malaise est dû au geste de Lucien qui cherche à l'exploiter ou encore aux effets propres aux montagnes russes.

La représentation de la langue française comporte également quelques aberrations, lesquelles ont été, à mon avis, planifiées par Wietz. À Berlin-Ouest, c'est la pièce «Gloire» de Lucien que Nais montera sur les planches de son théâtre. Si Lucien Chardon aspirait réellement à la gloire, les ambitions du Lucien de Wietz sont situées dans un univers encore plus mondialisé : "Mein Theaterstück "Gloire" ist ganz grosses Weltheater! Es wird Teil der Weltliteratur werden! $»^{8}(V I: 10)$ Pendant une année entière, la pièce de Lucien connaît un succès monstre à travers la République fédérale, ayant bénéficié de la caution du journal de type tabloïd Bild. Pour cette pièce, Lucien obtiendra même le «Prix mondiale » de littérature. Wietz écrit bel et bien l'adjectif « mondial » avec un « e »; à mon avis, cette erreur grammaticale a été intégrée de manière délibérée. Pour la réception de son prix, Lucien devra se rendre à Bangkok, alors qu'il est pourtant extrêmement endetté. Dans l'univers mondialisé que Wietz met en scène, il n'est pas insensé de faire le long vol pour récupérer un prix qui pourtant sera saisi par le ministre des finances Wolfgang Schäuble - en personne et à l'aéroport - dès que Lucien refoulera le sol allemand, en raison de l'importance des dettes fiscales de ce dernier (VI : 18). Les frontières n'existent plus, pas même celle du sceau de la qualité artistique, Lucien obtenant pour cette pièce sans doute médiocre - c'est forcément ce qui est suggéré quand on évoque le soutien que les «critiques

8 "Ma pièce de théâtre "Gloire" est du très grand théâtre mondial! Elle fera partie de la littérature mondiale! » 
littéraires » du quotidien Bild ont fourni - un prix international de littérature. Dans cet univers mondialisé, Paris aurait pu faire figure de dernière " frontière », en tant que «méridien de Greenwich de la littérature » (Casanova 2019 [2002] : 289-296). Mais ce n'est pas le cas; en allant jusqu'à proposer un français impur, en réhabilitant pour ainsi dire la faute d'accord - le Prix mondiale, et l'on verra plus loin que le titre du roman de Balzac est également transcrit avec deux fautes -, Wietz fait justement une sorte de pied de nez à l'hypothèse d'une Paris incarnant la Littérature même, soi-disant capitale de l'univers littéraire mondial, qui serait capable de distribuer le capital symbolique aux parutions littéraires, même étrangères (Casanova 2019 [2002]).

Nul désir, chez Wietz, de canoniser qui que ce soit: l'idée même de littérature mondiale est plutôt ridiculisée, même si l'admiration pour Balzac demeure plus que manifeste. Se profile toutefois un point de vue qui sera repris dans le traitement wietzéen de l'Histoire, et en particulier dans son analyse du raffinement de la technique : la mondialisation et la prolifération des échanges, culturels notamment, n'ont pas mené à une meilleure prise sur le réel; au contraire, "nous avons échappé à la sphère référentielle du réel et de l’histoire » (Baudrillard 1992 : 12), d'où l'impossibilité de l'exercice d'un réel « jugement de valeur », en art comme dans divers domaines (Baudrillard 1997 : 199).

\section{Un entrelacs d'anachronies}

Le roman graphique de Wietz est structuré par un réseau d'anachronies; dans le cadre de cet article, ce terme est préféré à celui d'« anachronisme ». Ainsi, je me réfère aux suggestions de Jacques Rancière qui évoque l'aspect «antihistorique» du terme d'anachronisme, mentionnant que l'histoire se fait précisément lorsque 
les hommes ne «ressemblent » pas à leur temps, pour autant qu'ils agissent en rupture avec «leur » temps, avec la ligne de temporalité qui les met à leur place en leur imposant de faire de leur temps tel ou tel «emploi ». Mais cette rupture n'est elle-même possible que par la possibilité de connecter cette ligne de temporalité à d'autres, par la multiplicité des lignes de temporalité présentes dans « un » temps (Rancière $1996: 66$ ).

Rancière associe à l'anachronie une valeur-réseau, comme si la sortie du temps sur laquelle elle repose permettait d'établir un lien très productif entre diverses époques, condition nécessaire pour qui souhaite faire (ou interpréter) l'Histoire. Ainsi, chez Wietz, les lignes de temporalité ne s'entremêlent pas uniquement sur le modèle de la dualité, mais aussi, fréquemment, sur le modèle de la pluralité. La référence intertextuelle sert également la réécriture wietzéenne de l'Histoire sous la forme d'un réseau d'anachronies : par exemple, dans la première partie dont les événements se déroulent en 1989-1990, lorsque le baron de Nucingen surgit, ce dernier fait lui-même clairement référence aux accords de Bâle III (VI :19), ces propositions de 2010 pour mettre en place des réglementations bancaires internationales à la suite de la crise des subprimes; trois époques distinctes sont ainsi liées au sein d'une seule bulle de parole.

Les anachronies les plus spectaculaires se situent toutefois dans la seconde partie du roman graphique. S'étant conduit exactement comme ceux qui l'avaient auparavant exploité, c'est-à-dire ayant réussi à encaisser des redevances auprès de plusieurs producteurs indépendants auxquels il avait promis la même série, Lucien se fait congédier à la fin de la première partie. Alors qu'il tente de mettre sa montre à l'heure d'hiver, il recule le temps, par mégarde, jusqu'à celui de vingt ans auparavant, ce qui donne lieu à un saut temporel gigantesque qui conduit à la seconde partie de l'œuvre, laquelle se déroule ainsi en 1969-1970, dans la République démocratique allemande. D'abord commentateur de films russes, Lucien connaît cependant le nouveau directeur de la télévision de la RDA; grâce à ce contact, il est embauché comme directeur de la programmation de la 
nouvelle chaîne de la DDR, renommée la chaîne DDR 2.0. Il faut noter d'emblée que la RDA imaginée par Wietz est constamment projetée dans l'avenir; les années 1969-1970 se confondent par moments avec l'ère post-réunification, et intègrent même des événements qui se sont produits dans la décennie 2010. Cependant, un certain ancrage principal en 1969 est de toute évidence maintenu; ainsi, l'on fait clairement allusion à la fondation de la deuxième chaîne de télévision de la RDA, Deutscher Fernsehfunk DFF 2, qui avait eu lieu en octobre 1969 pour célébrer les 20 ans de la fondation de la RDA : c'était alors la première fois que la RDA proposait une diffusion en couleurs. Renommer cette nouvelle chaîne $D D R$ 2.0, d'après l'appellation «Web 2.0 », constitue bien entendu un choix délibéré : il s'agit de privilégier l'anachronie pour faire de la RDA une société à la fine pointe de la technologie, ce qui n'a rien à voir avec la réalité historique. Les historiens évoquent ainsi le caractère nécessairement « défensif » (Barck, Classen et Heimann 1999 : 220) de la pratique médiatique dans la RDA, ainsi que la lutte pour l'information entre la RDA et la RFA comme l'élément qui fonde l'unicité du paysage médiatique est-allemand par rapport à celui des autres États communistes européens pendant la Guerre froide :

From the beginning, competition between the Federal Republic and the GDR was carried out on the air. During the Cold War both sides sought to win over the population of the other side and to discredit their "opponents" by often highly exaggerated propaganda claims. Nevertheless, the West soon gained an advantage, while East German media were increasingly forced into the defensive. This defensiveness not only characterized the provision of the necessary technical infrastructure, but also forms of programming and their conceptualization, as well as program content. The government was repeatedly forced to mobilize extra resources to stop Western stations from supplying the East with programming, or receiving better quality signals than GDR programs. The forced expansion of television in the East was also linked to previous developments in the Western zones. The GDR was continually behind - whether in broadcasting technology, the number of receivers in use, or in the implementation of new standards such as FM radio, stereo, or color 
television - and always trying to catch up with the "big brother" in the West who continued to set the standards (Barck, Classen et Heimann 1999 : 221) 9

À la lumière de ces faits, le portrait dressé par Wietz apparaît particulièrement fantaisiste. C'est en quelque sorte à travers son obsession pour l'Ouest et par le choix, jugé nécessaire, de réagir face aux contenus proposés par celui-ci que la télévision de la RDA a perdu de vue le réel même. Bien entendu, le journalisme dans la RDA n'était pas conforme aux attentes que l'on entretient à l'égard de celui qui est présent au sein des démocraties occidentales; mais on sait également que l'accès à la programmation ouest-allemande était très répandu à l'Est - pour la télévision, on parle de $60 \%$ de la population dès la fin des années 1950 et pour les années 1980, jusqu'à 80 \% (Barck, Classen et Heimann 1999 : 220). Et le plus grand nombre de ceux qui le pouvaient s'informaient en effet sur les chaînes de l'Ouest, faisant d'un tel geste une «habitude quotidienne » (Barck, Classen et Heimann 1999 : 220). Cela dit, même si Wietz opte pour une réécriture pour le moins étonnante de l'histoire des médias, on verra que le bédéiste évoque bien la course pour l'audience menée en RDA.

Plusieurs autres anachronies surgissent dans Verlorene Illusionen; je noterai notamment l'omniprésence des émissions de téléréalité en RDA. Ainsi, il faut trouver un roi adéquat pour la chaîne DDR 2.0, un «Super King » (VI: 47) qui

\footnotetext{
9 « Dès le début, la compétition entre la République fédérale et la RDA s'est exécutée à l'antenne. Pendant la Guerre froide, les deux côtés cherchaient à s'approprier la population de l'autre côté et à discréditer leurs "opposants" par des affirmations de propagande souvent hautement exagérées. Néanmoins, l'Ouest a rapidement acquis un avantage, pendant que les médias est-allemands étaient de plus en plus forcés à la défensive. Cette attitude défensive caractérisait non seulement la provision de l'infrastructure technique nécessaire, mais aussi les formes de programmation et leur conceptualisation, au même titre que le contenu des programmes. Le gouvernement a été forcé à plusieurs reprises de mobiliser des ressources supplémentaires pour empêcher les stations de l'Ouest de fournir de la programmation à l'Est, ou de recevoir des signaux de meilleure qualité que les programmes de la RDA. L'expansion forcée de la télévision à l'Est était aussi liée aux développements antérieurs dans les zones occidentales. La RDA était continuellement en retard - que ce soit dans la technologie de diffusion, le nombre de récepteurs en usage, ou dans la mise en place de nouveaux standards tels que la radio FM, le stéréo ou la télévision en couleurs - et elle tentait toujours de rattraper le "big brother" qui à l'Ouest continuait de fixer les standards ».
} 
devra aussi être «telegen » et « gut in HD rüberkommen »10 (VI : 44). Le conseil des ministres de la RDA, au sein duquel siège Mme Merkel, décide, pour trouver son roi, d'organiser un casting show, ces concours très représentés sous des formes de téléréalité depuis la fin des années 1990 tels que American Idol. Les téléspectateurs vont finalement élire le très célèbre footballeur Franz Beckenbauer à titre de nouveau roi de la RDA. Le roi élu deviendra pourtant rapidement un roi auquel on ment, précisément grâce aux médias et à la technologie très avancée dont est pourvue la RDA imaginée par Wietz. Dans une séquence précise, Franz Beckenbauer contacte le président déchu de la FIFA, Sepp Blatter, pour lui demander de laisser jouer le BFC Dynamo Berlin en Ligue des Champions: «Mein lieber Sepp, ich möchte gerne, dass du mir dabei hilfst, den BFC Dynamo Berlin in der Championsleague spielen zu lassen. Vielleicht kennst du ja einen Investor aus Katar, der sich an unserem ruhmreichen Verein beteiligen möchte? »11 (VI : 58) Blatter confie toutefois au directeur de la télévision de la RDA qu'il ne souhaite pas que le club de Berlin joue dans la Ligue des champions; le directeur choisit donc d'ériger un studio virtuel pour duper Franz Beckenbauer à huis clos en lui faisant croire que le BFC a bien rejoint la Ligue des champions. Une fausse partie est ainsi produite à l'écran et les voix des commentateurs sont trafiquées. Si le réseau d'anachronies qui est développé, notamment par la référence aux scandales récents entourant la FIFA, fait bien apparaître la corruption de plusieurs personnages haut placés dans le monde des médias de la RDA, c'est aussi un portrait du pouvoir pernicieux de la technologie actuelle que dresse Wietz, comme si le sempiternel progrès technique conduisait au contrôle démesuré - qui n'est pas très éloigné de celui qu'exerce une dictature, d'ailleurs -, au mensonge, et à la tromperie.

\footnotetext{
${ }^{10}$ Un « super roi » qui devra aussi être « télégénique » et « avoir une belle apparence en HD ».

11 «Mon cher Sepp, j'aimerais bien que tu m'aides à laisser jouer le BFC Dynamo de Berlin dans la Ligue des Champions. Peut-être connais-tu un investisseur du Qatar qui aimerait s'associer à notre club glorieux?»
} 


\section{Le moi privé - le moi public}

Afin d'augmenter les taux d'écoute de la chaîne DDR 2.0, son patron étant littéralement obsédé par ceux-ci, Lucien annonce qu'il souhaite justement « den Trend zum selbstreferentiellen Fernsehen verstärken $»^{12}$ (VI: 42) Pour ce faire, Lucien proclame qu'il embauchera des « von ARD und ZDF ausgemusterten Moderatorinnen ${ }^{13}$ (VI: 42), lesquelles pourront notamment «die DDR Zuschauer mit den Freuden einer gelungenen Darmspiegelung vertraut machen ${ }^{14}$ (VI: 42). On filme en effet une coloscopie plus loin dans le roman graphique : «Mit Hilfe einer Videokamera wird die Darmspiegelung per Livestream ausgespielt, so dass Sie und Ihre Familie live dabei sein können. Wer kein Tablet hat, kann sich eins bei der Charité ausleihen! $»^{15}$ (VI : 57). Wietz s'intéresse donc au partage immédiat des faits et gestes de tout un chacun que permettent les médias hypercontemporains, et à la très grande confusion entre les sphères publique et privée que celui-ci est susceptible d'induire. Tout n'est que représentation - on peut ici citer un extrait de Baudrillard tiré du Crime parfait :

Vivez votre vie en temps réel - vivez et souffrez directement sur l'écran. Pensez en temps réel - votre pensée est immédiatement codée par l'ordinateur. Faites votre révolution en temps réel - non pas dans la rue, mais dans le studio d'enregistrement. Vivez votre passion amoureuse en temps réel - avec vidéo incorporée tout au long de son déroulement. Pénétrez votre corps en temps réel - endovidéoscopie, le flux de votre sang, vos propres viscères comme si vous y étiez. Rien n'y échappe. Il y a toujours une caméra cachée quelque part. [...] On peut être appelé à rejouer tout cela devant n'importe quelle chaîne de télévision. [...]. On est sous le coup d'une retransmission instantanée de tous les faits et gestes sur n'importe quel canal. Jadis on aurait vécu cela comme contrôle policier. Aujourd'hui on le vit comme promotion publicitaire (Baudrillard 1995 : 46-47).

\footnotetext{
12 « renforcer la tendance à la télévision autoréférentielle »

13 « animatrices mises au rancart par ZDF et ARD ».

14 « rendre les téléspectateurs de la RDA familiers avec les plaisirs d'une coloscopie réussie ».

15 «Avec l'aide d'une caméra vidéo, la coloscopie sera rendue en livestream, pour que votre famille et vous puissiez y être en direct. Qui n'a pas de tablette peut en emprunter une à la Charité! »
} 
Baudrillard avait bien saisi, en 1995 déjà, des similitudes entre l'immense tutelle exercée par des sociétés qui sont fondées sur des mécanismes de surveillance, d'espionnage ou de contrôle renforcé telles que la RDA, et les possibilités ouvertes par les dernières avancées technologiques, lesquelles conservent de nombreuses traces de nos agissements, prisons étouffantes alors qu'elles devraient justement favoriser une plus grande liberté humaine. Il est clair que la mise en scène spectaculaire de soi, permise par le monde virtuel, demeure vide de sens chez Wietz. Ainsi, le contenu transmis à la télévision de la RDA reste, selon les aveux de Lucien et de son directeur, de qualité au mieux médiocre. Il s'agit de montrer au téléspectateur de la chaîne DDR 2.0, nous apprend Lucien, «wie er ohne es zu merken selbst Teil der Seichtheit des Programms wird »16 (VI: 42). Quant au directeur qui demande à Lucien d'en faire encore davantage pour augmenter les taux d'écoute de la télévision est-allemande, il pose la question: «Sollen wir etwa anspruchsvoll werden? ${ }^{17}$ (VI: 52), ce qui est peut-être une manière de rappeler le manque absolu de substance des émissions à contenu politico-idéologique qui étaient proposées dans la véritable RDA.

Exacerbé, narcissique à souhait, l'individualisme associé à la télévision autoréférentielle trouve toutefois sa contrepartie sur une page particulière $\mathrm{du}$ roman graphique, laquelle introduit des véritables traces de l'Histoire. Celles-ci sont relatives au slogan des fameuses manifestations du lundi de Leipzig: il s'agit bien entendu du slogan «WIR SIND DAS VOLK »18, qui apparaît en lettres majuscules rouges dans les toutes dernières pages du récit graphique $(V I: 66)$, figurant sur un monument dépouillé, simple poteau large aux teintes noire et grise foncée. À la case suivante est aussi représentée l'affiche «ICH BIN

\footnotetext{
16 «comment, sans le remarquer, il devient lui-même partie intégrante du caractère insipide du programme».

17 « devons-nous devenir exigeants, par hasard?»

18 « NOUS SOMMES LE PEUPLE ».
} 
VOLKER! »19, qu'un individu avait, lors des mêmes manifestations, fabriquée en guise de contrepoids; à nouveau, le lettrage de l'affiche est rouge et en lettres majuscules, ce qui est rare dans l'œuvre de Wietz (VI:66). Ce lettrage rappelle en fait le changement de la voix narrative qui surgit quatre pages auparavant. Alors que, le plus souvent, Verlorene Illusionen s'appuie sur une narration hétérodiégétique par le biais des récitatifs, et qui est en caractères noirs de surcroît, un «narrateur actorialisé » (Groensteen 2011 : 106) surgit en effet dans les toutes dernières pages, ce qui introduit une rupture sémiotique.

Ainsi, à la page 62, on trouve une bulle de pensée de Lucien : "Wo sind unsere Zuschauer von DDR 2.0 in SECAM und HD geblieben? Wo sind die letzten Zuschauer? $\gg^{20}(V I: 62)$ La réponse à ces questions, que l'on peut associer au surgissement d'un autre type de narrateur dans le roman graphique, est inscrite en lettres rouges sur un écriteau au fond beige, lequel est lui-même un élément du décor, placé sous une fenêtre au rez-de-chaussée d'un édifice résidentiel délabré : «Wir sind umgezogen! NACH DRÜBEN»²1 (VI: 62) Les mots «nach drüben » sont même en lettres majuscules, rapprochant ainsi ces paroles spectrales, à la fois par leur format et par leur couleur, des inscriptions «WIR SIND DAS VOLK» et «ICH BIN VOLKER!». Groensteen utilise l'expression «narrateur actorialisé » pour décrire «le cas de la narration à la première personne, par un personnage impliqué dans l'histoire et représenté graphiquement» (Groensteen 2011: 106). Or, si les personnages qui s'apparentent ici à un narrateur actorialisé s'exprimant au «nous », ces extéléspectateurs de la chaîne DDR 2.0, ne sont pas représentés concrètement sur le plan du corps, ils le sont bien de manière spectrale et conservent ainsi une présence essentielle dans le roman graphique, étant constamment évoqués par les

\footnotetext{
19 « JE SUIS VOLKER! ».

20 «Où sont demeurés nos téléspectateurs de DDR 2.0 en SECAM et HD? Où sont les derniers téléspectateurs?»

${ }^{21}$ « Nous avons déménagé! DE L'AUTRE CÔTÉ ».
} 
dirigeants de la chaîne de télévision qui aspirent à les identifier et à empêcher leur disparition complète. Je soulignerais également le dépouillement et le caractère direct du propos du narrateur actorialisé, qui lui confèrent une valeur plus factuelle qu'émotive; le fait que le lettrage employé pour présenter son propos soit identique à celui qui est utilisé, quatre pages plus loin, pour intégrer les slogans historiques des manifestations du lundi accentue également la valeur de référentialité de l'affirmation selon laquelle ces spectateurs ont déménagé à l'Ouest - fût-ce symboliquement, en s'informant désormais uniquement sur les chaînes de l'Ouest. Alors que le pacte de référentialité est le plus souvent mis à mal dans Verlorene Illusionen, l'appui sur des éléments historiques véritables étant déconstruit par les très nombreuses anachronies, les deux phrases qui sont sous la responsabilité du narrateur actorialisé réintroduisent une certaine pétition de fiabilité dans le traitement de l’Histoire.

Suivant une telle lecture, la recontextualisation des objets historiques tirés par Wietz des manifestations en ex-RDA se révèle indispensable. C'est que l'individualisme auquel l'affiche «ICH BIN VOLKER! » fait référence n'est pas une présence narcissique au monde comme celui qui est associé, dans l'œuvre graphique, à la technologie hypercontemporaine. Il s'agirait ainsi pour le bédéiste de désigner l'importance de réfléchir à l'avènement d'autres formes d'individualisme: un individualisme qui serait intéressé par l'engagement politique, par exemple. Car le «Volker» qui a élevé son affiche s'est bien rendu, après tout, aux manifestations du lundi; il n'est pas complètement coupé de la sphère politique. Dans un article paru en 2009 dans la taz (Tageszeitung), Dirk Knipphals rappelle à quel point la journée du 9 novembre 1989 a d'emblée été perçue comme un événement collectif : même si le journaliste reconnaît les aspects foncièrement positifs de la chute du mur, il s'interroge aussi, de fort intéressante manière, sur le fait que, ce jour-là, «das Verhältnis von Individuellem 
und Kollektivität [...] zu eindeutig in Richtung Kollektivität ausfällt »22 (Knipphals 2009 : 33). Évoquant précisément l'affiche "Ich bin Volker », véritable îlot brandi pendant les manifestions ayant mené à la chute de la RDA alors que l'on scande «Wir sind das Volk», il précise qu'opter pour un tel geste n'était pas que «lustig und entlastend» comme on a d'abord pu le croire, mais «von höherer Wahrheit » ${ }^{23}$ (Knipphals 2009 : 33). Les représentations médiatiques de la chute du mur qui ont fait le tour de la planète auraient-elles privilégié l'expérience de l'ensemble, et négligé, dans une certaine mesure au moins, l'expérience individuelle dans ses particularités? La question mérite d'être posée. Au regard de ces considérations, j'avancerais que, dans l'univers de Wietz, où les haut placés de la télévision croient qu'en proposant des formes de téléréalité, «die Zuschauerbindung wird 100\% betragen, da der Faktor der Selbstbespiegelung ebenfalls $100 \%$ ist $»^{24}(V I: 66)$, les prises de position tirées de l'histoire réelle de la République démocratique allemande rappellent l'urgence de réfléchir au rapport entre l'individu et la collectivité à notre époque, tension qui peut être associée à l'effondrement du régime communiste.

\section{La scène de clôture. Observations conclusives}

Je terminerai en présentant la chute du roman graphique: Lucien et ses collègues partent concrètement à la recherche du tout dernier téléspectateur de leur chaîne, faisant appel à la Nationale Volksarmee et aux services secrets. L'on arrive enfin à localiser celui-ci à Berlin-Est; Lucien et ses collègues se rendent chez le dénommé Huhlebeck en hélicoptère. Ce dernier a pu être repéré «an seiner terrestrischen Antenne », et il n'a ni le câble ni Internet; on pense même qu'il

\footnotetext{
${ }_{22}^{2}$ « la relation entre l'individu et la collectivité se tourne trop indiscutablement dans la direction de la collectivité ».

${ }^{23}$ « amusant et soulageant »; « d'une vérité supérieure ».

24 « Le lien avec le téléspectateur va s'élever à $100 \%$, car le facteur de l'observation narcissique de soi sera également à $100 \%$ ».
} 
aurait «noch eine Ziege im Stall $»^{25}(V I: 65)$. Alors que Lucien et ses collègues souhaitent «ihn [...] überzeugen in seinem Haus ein Studio einzurichten $»^{26}$ (VI: 65) pour réaliser une émission de téléréalité autour de sa personne, et ainsi remonter les taux d'écoute de la chaîne, Huhlebeck, tout comme nombre de ces « héros » de la téléréalité des vingt dernières années, est l'individu le plus ordinaire qui soit, qui vit également de l'aide sociale. Par contre, à la surprise générale, l'homme ne se trouve pas à son domicile lorsque les journalistes de DDR 2.0 s'y rendent : ces derniers découvrent plutôt des tablettes numériques ou des téléviseurs de poche. Sur les écrans de ceux-ci, on aperçoit Huhlebeck dans un fauteuil, lisant Illusions perdues de Balzac. Lucien s'en étonne, bien entendu, car on l'aurait «versichert, dass Huhlebeck nicht lesen und schreiben kann $»^{27}$ (VI : 67). La toute dernière page du roman graphique, une splash page d'ailleurs, est extrêmement riche de significations, car elle présente Huhlebeck assis dans son fauteuil, lisant le roman de Balzac, des caméras le filmant de tous les angles; la scène est simultanément projetée sur plusieurs écrans dont certains sont réfléchis par des surfaces-miroir. Le lecteur remarque alors que le titre du roman de Balzac a été reproduit avec deux fautes sur sa couverture : Illusion perdu, alors qu' «Illusion » devrait être au pluriel et l'adjectif " perdu », au féminin pluriel. Il s'agit à mon avis d'un choix délibéré de Wietz; le personnage de Huhlebeck est d'abord maintenu du côté d'un certain analphabétisme.

Qu'en est-il aujourd'hui du rapport avec les grandes œuvres de la littérature mondiale, nous demande Wietz, si celui qui les tient dans ses mains est analphabète? Est-on toujours capable de les comprendre, ou tout rapport à la culture savante a-t-il été exterminé? Alors que notre univers est pourtant celui de l'accès à toute la culture, cet accès illimité n'est-il pas précisément la source de

\footnotetext{
25 " par son antenne terrestre »; « encore une chèvre dans l'étable».

26 « le convaincre d'installer un studio dans sa maison ».

27 « assuré que Huhlebeck ne sait ni lire ni écrire ».
} 
notre perte? Notre approche du monde, qui consiste à accumuler le maximum d'informations sur celui-ci grâce à la technologie, parfois au détriment de la qualité et à une vitesse effrénée, mènerait-elle à la déshumanisation, au devenirécran plutôt qu'à une meilleure prise sur le réel? Car Huhlebeck n'est pas passé à l'Ouest, ou ne s'est pas tourné vers les médias ouest-allemands, alors qu'on aurait pu s'attendre, eu égard à la réalité historique, à ce qu'une telle avenue soit au moins évoquée à la fin du roman graphique. Plutôt que d'inviter à une réflexion sur les médias qui ne déborderait que peu du cadre de l’Allemagne divisée, Wietz souhaite offrir un regard englobant sur les médias, toutes époques confondues : il existe un réel danger à tenter de rendre le monde efficace par les médias, à instrumentaliser le journalisme ou la littérature; qu'il s'agisse de préférer le nombre de téléspectateurs à la qualité de l'information transmise, de placer le médium au service de l'idéologie ou à celui de son portefeuille. Alors que Huhlebeck aurait pu être figuré par Wietz comme le dernier bastion d'un rapport plus naturel au monde - après tout, avant qu'on ne découvre son «devenir-écran », sa relation avec le monde était présentée comme très peu influencée par les nouvelles technologies, puisqu'il est décrit comme quelqu'un qui n'a ni le câble ni Internet, et qui posséderait «encore une chèvre dans l'étable »-, il n'en est rien. La capacité d'imaginer un autre rapport au monde ne se trouve même pas de ce côté, celui des déclassés sociaux pourtant si souvent contraints, au cours de l'Histoire, de faire preuve d'invention pour survivre. Huhlebeck a déjà disparu; s'il n'est pas passé à l'Ouest, il est néanmoins entré dans l'écran, il est irrécupérable. Le fait que le titre de la grande œuvre qu'il tient entre ses mains soit fort malmené ne rappelle cependant pas que l'analphabétisme du personnage, mais il évoque aussi la grande « esthétisation » (Baudrillard $1990: 24$ ) de notre monde.

Selon Baudrillard, la société contemporaine aurait élargi l'esthétisation à tous les objets, même aux objets pour ainsi dire anti-culturels, précisément «à 
travers la publicité, les médias, les images » (Baudrillard 1990 : 24). Huhlebeck, personnage anti-culturel s'il en est, lit Balzac; et celui qui trouverait matière à soulever le caractère improbable de cette représentation pourrait être taxé d'élitisme. Tout se dit, toute opinion peut être exprimée dans un monde qui, dans les arts mais également dans d'autres sphères, échappe aux critères du « beau » et du «laid». Si l'univers hypersophistiqué dans lequel Huhlebeck est plongé ne le prive pas, de manière théorique, d'un certain accès à la culture restreinte, il ne lui offre cependant pas les moyens de pénétrer véritablement une telle culture; le titre du roman de Balzac, grammaticalement incorrect chez Wietz, renvoie également à cette réalité. Alors que la télévision et Internet auraient pu devenir les instruments par excellence pour permettre à ceux qui n'ont pas eu un accès aisé à la culture de se fabriquer un autre destin, cela n'a pas été le cas selon le bédéiste.

Est-ce à dire que l'affiliation avec Balzac se voit désavouée à la fin du roman graphique? Je ne suis pas du tout de cet avis, bien que Wietz semble souligner à grands traits la difficulté de pénétrer les classiques à notre époque. Car la grande originalité de l'entreprise de Balzac, outre l'aspect totalisant de son analyse des réseaux littéraires et médiatiques, est d'avoir résolument situé cette analyse dans l'Histoire. Tout en décrivant les rouages d'un monde dans lequel « l'influence et le pouvoir du journal n'est qu'à son aurore » (Balzac 1981[18371843] : 321), Balzac anticipe également de manière précise l'avenir du journal :

Ainsi, tous les journaux seront dans un temps donné, lâches, hypocrites, infâmes, menteurs, assassins; ils tueront les idées, les systèmes, les hommes, et fleuriront par cela même. Ils auront le bénéfice de tous les êtres de raison : le mal sera fait sans que personne en soit coupable (Balzac 1981[1837-1843] : $322)$.

Dans un roman graphique qui décrit un monde en voie de devenir purement virtuel, où l'homme en tant qu'être foncièrement humain, désirant et doté d'un 
corps semble voué à la disparition, il n'est ainsi pas étonnant que Balzac fasse figure de référent ultime. La leçon de Wietz est sans doute l'incitation à revenir vers des identités fortes («Ich bin Volker») face au danger que représente l'intelligence artificielle absolue, entreprise difficile dans notre monde où une panoplie de modèles identitaires sont proposés, retransmis en permanence dans les médias jusqu'à nous abrutir. Il est ainsi révélateur que la pétition de vérité historique soit transmise par une voix narrative qui n'est que spectrale, et que le Lucien qui dans la première partie commence à exploiter ses semblables soit placé dans l'univers anhistorique que constitue la fête foraine franco-allemande, dans un non-lieu et hors du temps, entre les cultures sans appartenir vraiment à l'une d'entre elles.

C'est bien une certaine difficulté d'habiter l'histoire à notre époque que décrit Wietz; et l'on pourrait aussi voir dans l'importance attribuée au slogan «Wir sind das Volk» et à l'affiche «Ich bin Volker! », des références à l'émergence du populisme de droite dans l'Allemagne de l'Est au cours de la dernière décennie. À rebours de la radicalisation de l'AfD, dont la relativisation, par exemple, des crimes de la période nazie par Alexander Gauland a plutôt constitué un vrai refus de l'Histoire, et alors même que le fantasme du « peuple » est sollicité par les nouveaux nationalismes européens, l'allusion à l'affiche «Ich bin Volker! » évoquerait le goût de l'indépendance d'esprit et de la réflexion individuelle, obstinée et critique. Elle désignerait un individualisme qui vise avant tout le développement des responsabilités de l'individu pour faire face, audelà du seul rapport à la technologie, aux grands enjeux de notre époque. 


\section{Bibliographie}

Abel, J. et C. Klein. (2016). Comics und Graphic Novels. Eine Einführung. Stuttgart, J. B. Metzler.

Augé, M. (1992). Non-lieux, introduction à une anthropologie de la surmodernité. Paris, Seuil.

De Balzac, H. (1981[1837-1843]). Illusions perdues. Paris, Gallimard.

Barck, S., C. Classen et T. Heimann. (1999). «The Fettered Media. Controlling Public Debate ». Dans K. Jarausch (dir.), Dictatorship as Experience. Towards a Socio-Cultural History of the GDR. New York et Oxford, Berghahn, p. 213239.

Baudrillard, J. (1997). Écran total. Paris, Galilée.

Baudrillard, J. (1995). Le crime parfait. Paris, Galilée.

Baudrillard, J. (1992). L'illusion de la fin. Paris, Galilée.

Baudrillard, J. (1990). La transparence du mal. Paris, Galilée.

Casanova, P. (2019 [2002]). "Paris, méridien de Greenwich de la littérature ».

Dans C. Charle et D. Roche (dir.), Capitales culturelles, capitales symboliques :

Paris et les expériences européennes (XVIII ${ }^{e}-X X^{e}$ siècles). Paris, Éditions de la Sorbonne, p. 289-296.

Groensteen, T. (2011). Bande dessinée et narration. Système de la bande dessinée, vol. 2. Paris, PUF.

Knipphals, D. (2009). «Tag der deutschen Differenz ». taz (Die Tageszeitung), 7 novembre, p. 33.

McCloud, S. (1993). Understanding Comics. The Invisible Art. New York, Harper Collins.

Rancière, J. (1996). "Le concept d'anachronisme et la vérité de l'historien ». $L^{\prime}$ inactuel : psychanalyse $\mathcal{E}$ culture, $\mathrm{n}^{\circ} 6$, p. 53-68. 
Schmitz-Emans, M., en collaboration avec C. A. Bachmann. (2012). LiteraturComics. Adaptationen und Transformationen der Weltliteratur. Berlin et Boston, De Gruyter.

Wietz, H. (2017). Verlorene Illusionen. Berlin, Walde + Graf. 